\title{
Production of Biodiesel from Vegetable Oils \\ ${ }^{* 1}$ Pampari Shailaja, ${ }^{2}$ Bajireddy Vinitha \\ M.Sc. Biotechnology, Department of Biotechnology, University College of Science Osmania University, Saifabad, Hyderabad-500004 \\ Email: pamparishailaja12@gmail.com,vinithabsgb@gmail.com
}

\section{Received: $10^{\text {th }}$ Feb 2018, Accepted: $18^{\text {th }}$ March 2018, Published: $3^{\text {th }}$ April 2018}

\begin{abstract}
Biodiesel is a biofuel used alternative to conventional diesel fuel majorly used in order to reduce greenhouse gases, non-sulphur emissions and non-particulate matter pollutants. This article reviews the production of biodiesel from various refined vegetable oils like sunflower oil, groundnut oil, coconut oil, cotton oil, and mustard oil and protocol standardization for biodiesel production from waste vegetable oil. Extraction of biodiesel from vegetable oil undergoes chemical processing called transesterification. Presence of free fatty acid is tested by dissolving vegetable oil in isopropyl alcohol and ph indicator. After sample collection the vegetable oil undergoes preliminary treatment in case of waste vegetable oil in order to remove any debris or reminants from the oil. Later transesterification reaction takes place wherein oil reacts with alcohol (methanol) and catalyst (Naoh or Koh). Waste vegetable oil is added to catalyst and methanol in the container and is stirred well for complete dissolution. After 12- 24 hours, 2 clear phases can be observed with a clear interphase differentiating crude biodiesel and glycerin. Further the crude biodiesel is separated by different separation techniques and is further processed for washing using warm water. In order to avail pure biodiesel the washing step is carried out for 2-3 times. Further confirmation tests are carried out quantitatively and qualitatively. Different volumes of crude biodiesel were obtained from different oils used. Quantity of the crude biodiesel produced depends on the catalyst used.
\end{abstract}

Keywords: biofuel, vegetable oils, transesterification, catalyst, production

\section{Introduction}

Fuels generated from biological feed stocks are termed as bio fuels. Alternative fuels have gained recent significant attention. As the calorific value of vegetable oils is comparable to that of diesel, they could be used as fuels in compression ignition engines. Due to the predicted shortness of conventional fuels and environmental concerns, a search for Fuels is of great importance because they can be burned to produce significant amounts of energy. Renewable and clean alternative fuels have received increasing attention for current and future utilization. Fatty acid methyl esters (FAME) show great potential as diesel substitutes, and they are known to be sources of biodiesel. Biodiesel (FAME) is a clean-burning fuel derived from vegetable oils or animal fat and is an advantageous alternative to fossil diesel fuel because of its biodegradability, biorenewable nature, very low sulfur content and toxicity, low volatility or flammability, good transport and storage properties, higher cetane number, and its salutary atmospheric $\mathrm{CO}_{2}$ balance for production. Currently, more than $95 \%$ of the world biodiesel is produced from edible oil which is easily available on large scale from the agricultural industry. The use of non-edible oil for biodiesel production compared with edible oil is very significant in developing countries. Moreover, biodiesel returns about $90 \%$ more energy than the energy that is utilized to produce it .Biodiesel mixed with conventional diesel in some proportions can be used to run any existing conventional compression ignition engine and does not require any modifications to be done to the engine .Due to benefits like renewable in nature, low cost and green house gas reduction potential, biodiesel is nowadays incorporated all over the world. Biodiesel can be processed from Thermal cracking of vegetable oils and Transesterification. Transesterification is the most common process, in this process an ester compound is exchanged by an alcohol in the alkyl group. Transesterification of triglycerides (vegetable oils) with alcohol and suitable catalyst is carried out. Biodiesel can be produced from different triglyceride sources such as vegetable oils (that can be edible, non-edible or waste oils), animal fats (mostly edible fats or waste fats) and microalgae oil. Economic feasibility of biodiesel depends on the availability of low-cost feed stocks. Waste cooking oil is a cost effective and promising feed stock . WCO with higher FFA content results is decrease in the overall yield. In this case, esterification is to be done before Transesterification. The most relevant process parameters in these kinds of operation are reaction temperature, ratio of alcohol to vegetable oil, amount of catalyst, mixing intensity (RPM), catalyst, and the raw oils used. In contrast, ester exchange is a more advanced method. The triglyceride can be transformed into monoester. Due to the transesterification in the ester exchange process, the viscosity of vegetable oil is reduced and heat values maintained. The long- and branchedchain triglyceride molecules are transformed to mono-esters and glycerin. Commonly-used shortchain alcohols are methanol, ethanol, propanol and butanol. Methanol is used commercially because of its low price. Because this process is a reversible 
reaction, the output of biodiesel will be directly influenced by the proportion of reactants, the type and the dosage of the activator, and the reaction conditions. Biodiesel offers the promise of numerous benefits related to energy security, economics, expansion of the agriculture sector and reduction of pollutant emission.

\section{Materials and Methods}

Sample: vegetable oil that has been repeatedly used for cooking i.e. used cooking oil (uco) is collected. Defined quantities of sunflower oil, groundnut oil, coconut oil, cotton oil, and mustard oil are collected. Filtration/preliminary treatment of oils: waste vegetable oil that has been collected is filtered using filter paper in a conical flask. All the waste present in the oil are settled on the filter paper and filtered oil is collected in the conical flask drop wise. Filtration of waste vegetable oil takes longer time. Duration of filtration process depends on quantity of oil used.

Mixing: $0.40 \mathrm{gm}$ of catalyst i.e., $\mathrm{NaoH}$ or $\mathrm{KoH}$ is measured quickly into a conical flask in order to avoid evaporation of the catalyst. To that $10 \mathrm{ml}$ of methanol is added. The conical flask is allowed to stir until the catalyst is completely dissolved. This mixture is now called as lye. Now to the above mixture $40 \mathrm{ml}$ of waste vegetable oil is added and is stirred well for complete dissolution. Naoh takes longer time to dissolve when compared with $\mathrm{KoH}$. Then the above taken conical flask along with the mixture is covered with a aluminium foil or PET bottles can be used in order to use lids to close. Then this mixture is manually stirred in water bath or placed on a magnetic stirrer with temperature less than 60degrees. Continuous Stirring should be carried out for 60 minutes. Allow the mixture to settle overnight. Care should be taken in order to avoid the mixture from atmosphere. It takes 12-24 hours for the reaction to take place. air tight bottles should be used or tightly packed with foil is recommended.

Trans esterification: After 12- 24 hours, 2 clear phases can be observed with an clear interphase. After the trans esterification reaction takes place crude oil formed is seen as the top layer and glycerol settles at the bottom which can be further processed to produce

soap.

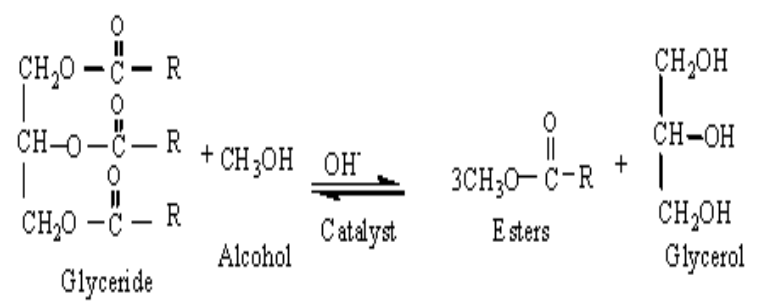

Separation: After the appearance of two phases, decant the upper crude biodiesel into another conical flask or beaker. Do not mix the two layers. The thus obtained crude biodiesel can further be processed for washing.

Washing: crude biodiesel contains contaminants such as soaps, excess methanol, residual catalyst and glycerol. Hence the crude biodiesel is purified with lukewarm water. Add $10 \mathrm{ml}$ of lukewarm water to the separated crude biodiesel and allow it to settle for good 30min. Time duration depends on the amount of oil taken. After $30 \mathrm{~min}$ you can see 2 separate layers with slightly purified biodiesel and the remaining excess is settled below. Washing procedure is carried out 2-3 times in order to get purified oil. Bubble wash method or stir washing method can be used. After the washing procedure is completed separate the top layer I.e., purified biodiesel and it is further processed for drying.

Fig 1: Different Oils used for the Production of BioDiesel

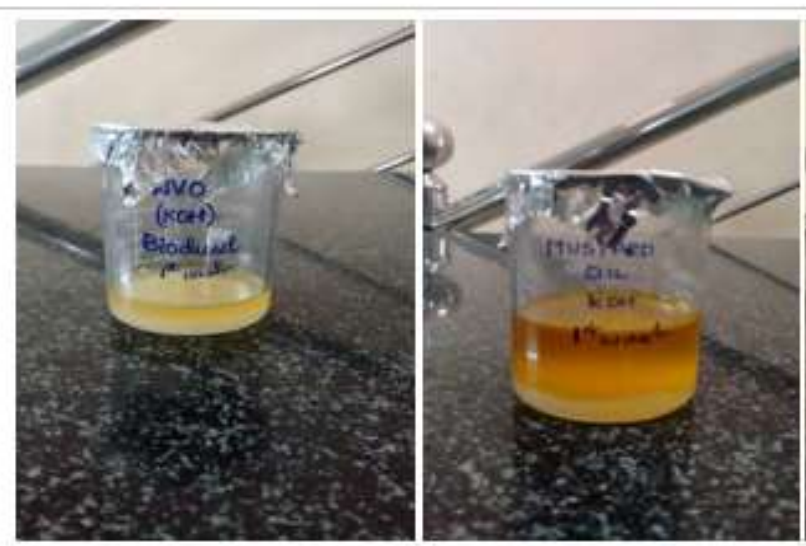

The above mentioned pictures show the washing of waste vegetable oil, mustard oil, coconut oil and cotton oil using warm water

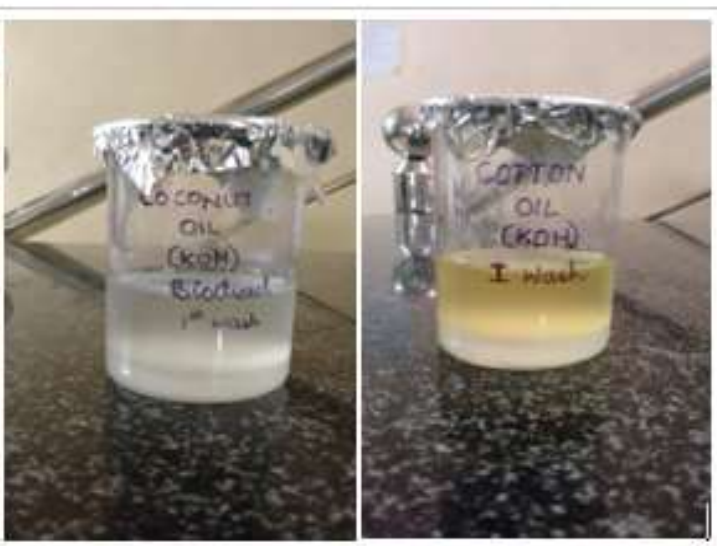

Drying: $0.5 \mathrm{gm}$ of $\mathrm{Na} 2 \mathrm{so} 3$ is added in order to remove any water content present in the biodiesel. Further quantitative and qualitative analysis of the purified biodiesel is carried out which include 
volume, mass, volume and $\mathrm{pH}$ of the obtained biodiesel is checked for confirmation.

\section{Results and Discussion}

Further mass, pH (aqueous ph is checked by adding 5 drops of biodiesel to $1 \mathrm{ml}$ of water) and volume of the various refined oils and waste cooking oil is determined.

\section{Fig 2: Esterification of Sunflower oil}

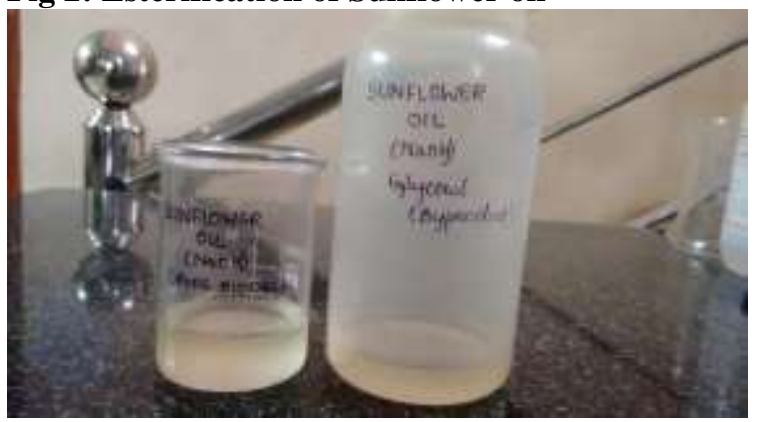

The picture represents separated crude biodiesel and glycerin from sunflower oil using catalyst $\mathrm{NaOH}$

Biodiesel produced by using catalyst $\mathrm{KOH}$ is measured which has Mass: $9.06 \mathrm{gm}$; volume: $18 \mathrm{ml}$; aqueous ph: 8

Biodiesel produced by using catalyst $\mathrm{NaOH}$ is measured which has mass: $13.02 \mathrm{gm}$; volume: $24 \mathrm{ml}$ aqueous ph: 8

Groundnut oil: Not suitable for production of biodiesel as it gets solidified during trans esterification reaction

Fig 3: Esterification of Cotton oil with $\mathrm{NaOH}$

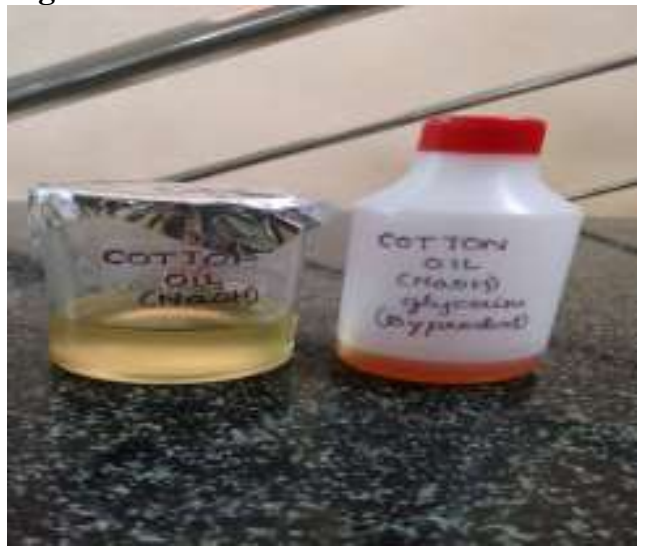

Fig 3: Esterification of Cotton Oil with $\mathrm{KOH}$

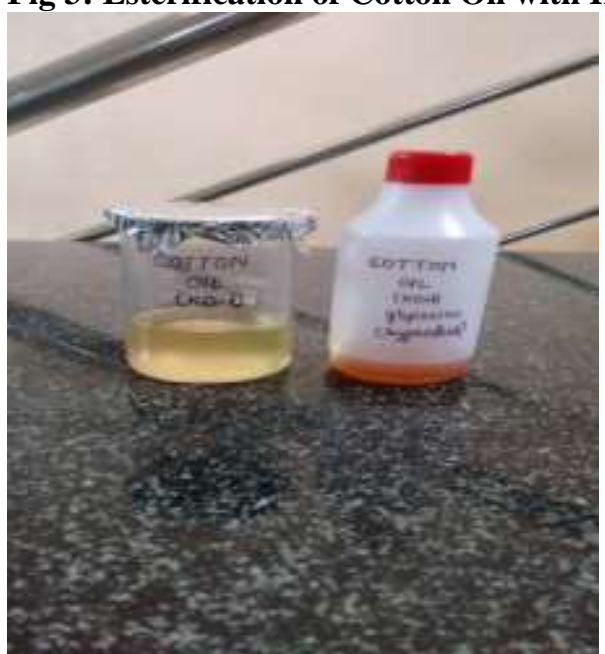

The above picture represents separated crude biodiesel and glycerin from cotton oil using Naoh and Koh catalyst

Biodiesel produced by using catalyst $\mathrm{KoH}$ is measured which has

Mass: $18.4 \mathrm{gm}$; volume: $22 \mathrm{ml}$;aqueous ph: 7

Biodiesel produced by using catalyst $\mathrm{NaOH}$ is measured which has

mass: $22.4 \mathrm{gm}$; volume : $27 \mathrm{ml}$; aqueous ph : 7

\section{Fig 4: Esterification of Coconut oil with $\mathrm{NaOH}$} and $\mathrm{KOH}$
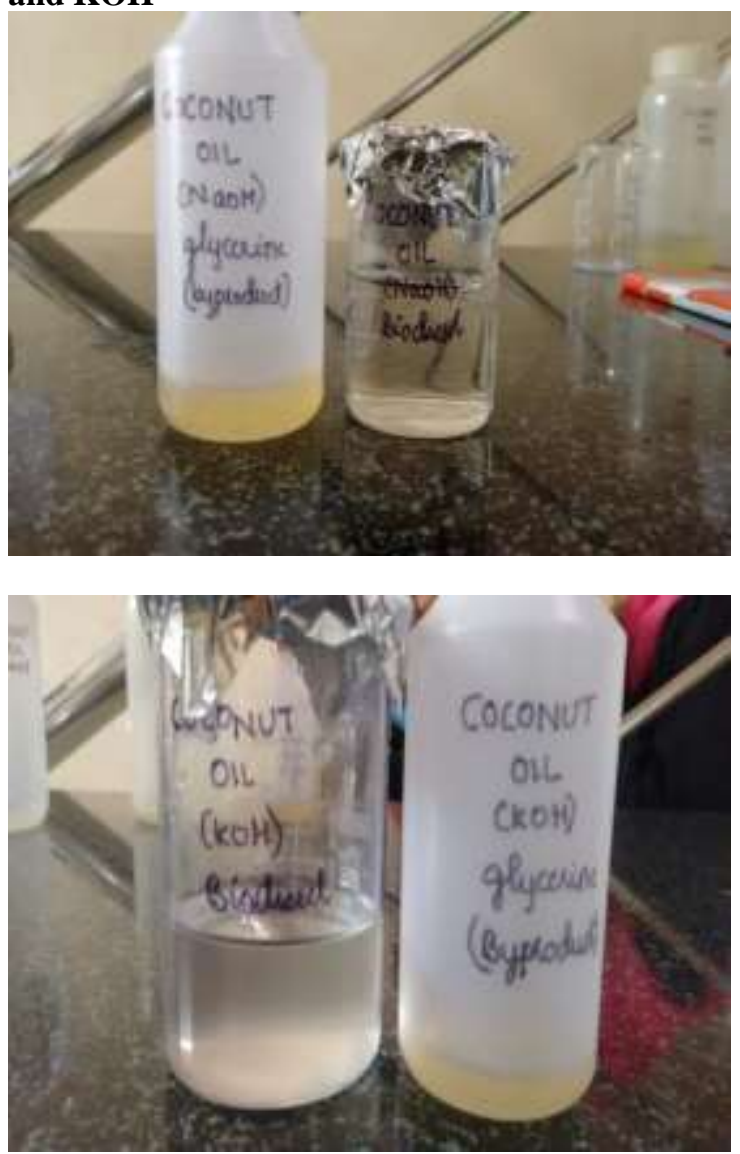
The above picture represents separated crude biodiesel and glycerin from coconut oil using $\mathrm{NaOH}$ and $\mathrm{KOH}$

Biodiesel produced by using catalyst $\mathrm{KOH}$ is measured which has

mass: $19.88 \mathrm{gm}$; volume:24ml ; aqueous ph: 7

Biodiesel produced by using catalyst $\mathrm{NaOH}$ is measured which has

mass:22.04gm ; volume: $27 \mathrm{ml}$; aqueous ph: 7

Fig 5: Esterification of Mustard oil with $\mathrm{NaOH}$ and $\mathrm{KOH}$ :
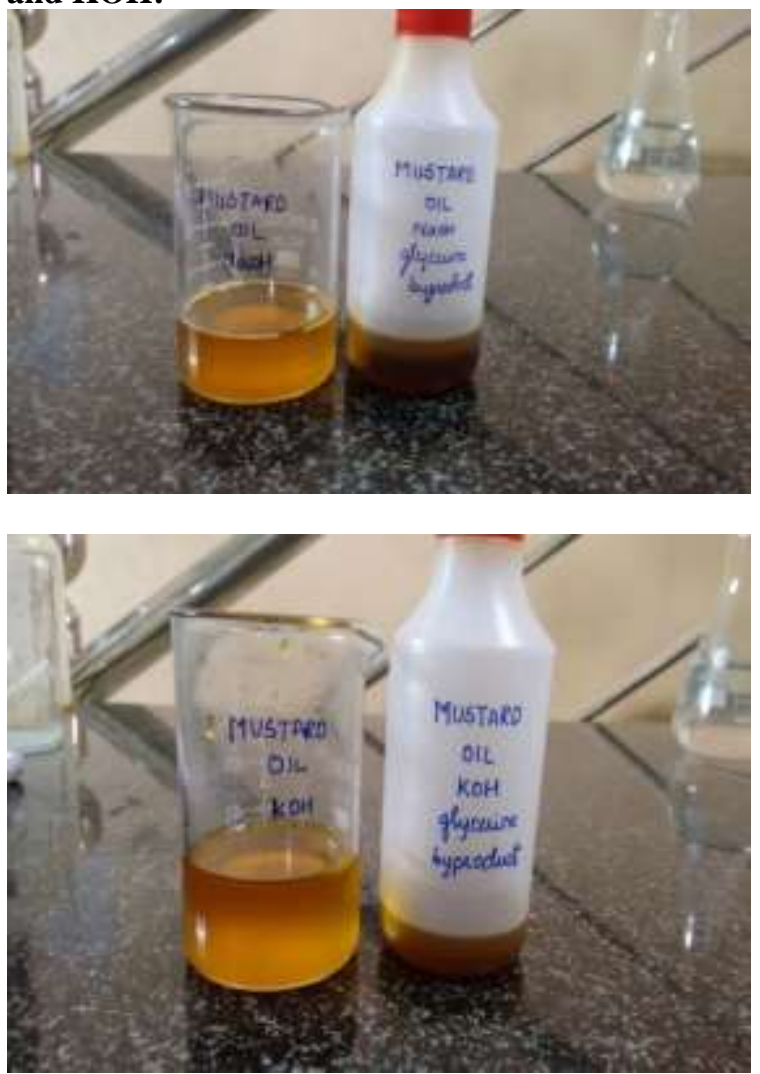

The above picture represents separated biodiesel and glycerin from mustard oil using $\mathrm{NaOH}$ and $\mathrm{KOH}$

\section{Biodiesel produced by using catalyst $\mathrm{KoH}$ is} measured which has

mass:25.53 gm ; volume:30ml ; aqueous ph: 7

Biodiesel produced by using catalyst $\mathrm{NaOH}$ is measured which has

mass:16.43gm ; volume:20ml ; aqueous ph : 7
Fig 6: Esterification of Waste vegetable oil with KOH:

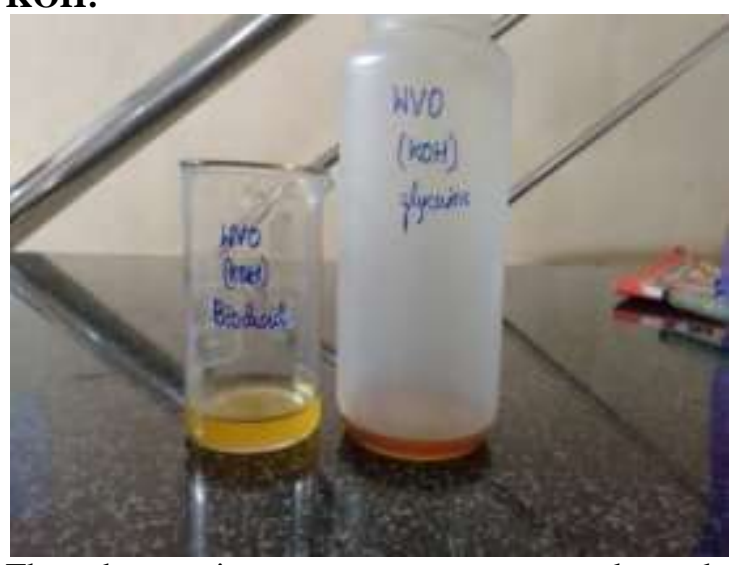

The above picture represents separated crude biodiesel and glycerin from waste vegetable oil using catalyst $\mathrm{KOH}$

Biodiesel produced by using catalyst $\mathrm{KOH}$ is measured which has

mass: 9gm ; volume: $18.4 \mathrm{ml}$; aqueous ph:7

Biodiesel produced by using catalyst $\mathrm{NaoH}$ is measured which has

mass:11.2gm ; volume: $20 \mathrm{ml}$; aqueous ph:7

\section{Conclusion}

In order to reduce the usage of harmful fuels biodiesel can be promisingly used to protect the environment. In this procedure methanol is used with catalyst Noah or $\mathrm{KoH}$ All the vegetable oils yielded good amount of biodiesel when treated with Noah rather than $\mathrm{KoH}$. Hence Noah can be dominantly preferred in order for production of biodiesel from refined vegetable oils or waste vegetable oils. But mustard oil has different result as it yielded more amount of biodiesel when treated with $\mathrm{KoH}$ rather than Noah. Waste vegetable oil yielded good amount of biodiesel when treated with Noah. Usage of either virgin oils or waste vegetable oils had almost similar results and approximately similar amount of biodiesel production. But waste vegetable oils are preferred in order for their proper usage and further processing. Processed vegetable oil to biodiesel will not effect the engine and as well as protects the environment from harmful gases. Hence waste vegetable oils can be efficiently used for production of biodiesel.

\section{References}

1) Mustafa B., "Potential alternatives to edible oils for biodiesel production - a review of current work," Energy Conversion and Management, 52(2), 14791492, 2011.

2) Arjun B.C., Martin, S.T., Suzanne, M.B., Chris, K.W. and Rafiqul, M.I., "Non-edible plant oils as new sources for biodiesel 
production," International Journal of Molecular Science, 9(2), 169-180, 2008.

3) M. Thirumarimurugan, V. M. Sivakumar, A. Merly Xavier, D. Prabhakaran, and T. Kannadasan;;International Journal of Bioscience, Biochemistry and Bioinformatics, Vol. 2, No. 6, November 2012.

4) Colin J. Stacy, Cory A. Melick, Richard A. Cairncross; Fuel Processing Technology 124 (2014) 70-77.

5) Gabriel O. Ferrero , Manuel F. Almeida, Maria C.M. Alvim-Ferraz, Joana M. Dias; Energy Conversion and Management 89 (2015) 665-671.

6) Sneha E. Mahesh, AnandRamanathan b, K.M. Meera S. Begum , Anantharaman Narayanan ;:Energy Conversion and Management 91 (2015) 442-450 